\title{
Identification of ireA, 0007, 0008, and 2235 as TonB-dependent receptors in the avian pathogenic Escherichia coli strain DE205B
}

\author{
Zhonghua Zhang, Shan Jiang, Yun Liu, Yu Sun, Peixin Yu, Qianwen Gong, Hang Zeng, Yihao Li, Feng Xue, \\ Xiangkai Zhuge, Jianluan Ren, Jianjun Dai ${ }^{*}$ and Fang Tang* ${ }^{*}$
}

\begin{abstract}
Avian pathogenic Escherichia coli (APEC), a pathotype of extraintestinal pathogenic E. coli, causes one of the most serious infectious diseases of poultry and shares some common virulence genes with neonatal meningitis-associated E. coli. TonB-dependent receptors (TBDRs) are ubiquitous outer membrane $\beta$-barrel proteins; they play an important role in the recognition of siderophores during iron uptake. Here, in the APEC strain DE205B, we investigated the role of four putative TBDRs - ireA, 0007, 0008, and 2235-in iron uptake. Glutathione-S-transferase pulldown assays indicated that the proteins encoded by these genes directly interact with TonB. Moreover, the expression levels of all four genes were significantly upregulated under iron-depleted conditions compared with iron-rich conditions. The expression levels of several iron uptake-related genes were significantly increased in the ireA, 0007, 0008, and 2235 deletion strains, with the upregulation being the most prominent in the ireA deletion mutant. Furthermore, iron uptake by the ireA deletion strain was significantly increased compared to that by the wild-type strain. Moreover, a tonB mutant strain was constructed to study the effect of tonB deletion on the TBDRs. We found that regardless of the presence of ton $B$, the expression levels of the genes encoding the four TBDRs were regulated by fur. In conclusion, our findings indicated that ire A, 0007, 0008, and 2235 indeed encode TBDRs, with ireA having the most important role in iron uptake. These results should help future studies explore the mechanisms underlying the TonB-dependent iron uptake pathway.
\end{abstract}

\section{Introduction}

Avian pathogenic Escherichia coli (APEC), a pathotype of extraintestinal pathogenic E. coli (ExPEC), causes serious infectious diseases in poultry [1,2]. Different serotypes of APEC cause local or systemic infectious diseases in poultry, including respiratory infections, sepsis, polyserositis, coligranuloma, cellulitis, yolk sac infection, omphalitis, and swollen head syndrome, resulting in significant economic losses to the poultry industry [3]. Strains of APEC

*Correspondence: daijianjun@njau.edu.cn; tfalice@126.com MOE Joint International Research Laboratory of Animal Health and Food Safety, Key Laboratory of Animal Bacteriology, Ministry of Agriculture, College of Veterinary Medicine, Nanjing Agricultural University, Nanjing 210095, China and neonatal meningitis-associated E. coli (NMEC, a subpathotype of ExPEC), the latter of which causes infections in humans, reportedly share some common virulence genes [4-6]. It is thus particularly important to study the genes encoding virulence factors in APEC strains. These strains contain several virulence-associated genes that encode various virulence factors, including adhesins ( $i m C$, ompA, papC), invasins (ibeA), avian haemolysins $(h l y F)$, serum survival proteins (iss, ompT), and siderophores (iutA, fyuA, iroN); moreover, they show the presence of a pathogenicity island [7-9].

Iron is a vital micronutrient that regulates enzyme activity and metabolism. This element plays a key role in basic cellular processes, such as cellular respiration, 
DNA replication, and electron transport and is accordingly essential for bacterial survival in host tissues [10-12]. Furthermore, iron is a necessary growth factor for bacteria and is reportedly involved in the expression of bacterial virulence factors [13]. Iron uptake factors evidently play a pivotal role in $E$. coli growth and pathogenesis. Bacteria employ different strategies to absorb iron from their environment, including siderophore-mediated iron uptake, which occurs on the cell surface [14]. In addition, bacteria either excessively reduce the external $\mathrm{pH}$ or dissolve iron oxide to meet their iron requirements by reducing ferric iron to a relatively soluble ferrous form. Another common strategy is to synthesize and secrete iron chelators, such as siderophores, as intracellular iron $\left(\mathrm{Fe}^{2+}\right)$ is rarely found in natural conditions, and $\mathrm{Fe}^{2+}$ can be readily oxidized to $\mathrm{Fe}^{3+}$ in the presence of oxygen and water [15]. Siderophores then combine with the available iron $\left(\mathrm{Fe}^{3+}\right)$ to form an iron-siderophore complex, which binds to specific receptor proteins on the bacterial cell surface, consequently entering cells via the TonBdependent transport system, followed by iron release. Siderophore-mediated ferric uptake requires specific outer membrane $(\mathrm{OM})$ receptors, such as FhuA, FecA, and FepA, which reportedly exist in E. coli [16]. These OM receptors share the same structural properties and acquire energy by coupling with TonB proteins located in the inner membrane, and they are referred to as TonB-dependent receptors (TBDRs) [17].

TBDRs are known to actively transport ferric-siderophore complexes in Gram-negative bacteria, and they also transport diverse antibiotics, vitamins, nickel complexes, and carbohydrates [18-20]. Transporters involved in iron uptake have very strict siderophore selectivity. A strong correlation exists between the amount of iron and siderophores that bacteria can use and the number of genes encoding iron-regulated TBDRs [19]. In simple terms, iron depletion triggers the upregulation of genes encoding TBDRs.

In this study, upon analysing the whole genome of the APEC strain DE205B, we identified six putative TBDRs; however, the construction of two mutants failed. Thus, we eventually investigated the roles of four putative TBDRs-ireA, 0007, 0008, and 2235-in iron uptake.

\section{Materials and methods}

Bacterial strains, plasmids, and growth conditions

The bacterial strains and plasmids used in this study are listed in Table 1. The APEC strain DE205B (O2:K1), which was isolated from a duck with neurological symptoms, was previously determined to have no antibiotic (ampicillin, kanamycin, and chloramphenicol) resistance [21-25]. All the APEC strains used in this study were cultured in Luria-Bertani (LB, Oxoid, Thermo-Fisher,

Table 1 Bacterial strains and plasmids used in this study.

\begin{tabular}{|c|c|c|}
\hline Strain or plasmid & Characteristics & References \\
\hline \multicolumn{3}{|l|}{ Strain } \\
\hline DE205B & $\mathrm{O} 2: \mathrm{K} 1, \mathrm{NaIR}$ & {$[21-25]$} \\
\hline DE205B $\triangle$ ireA & ireA Deletion mutant strain & {$[21]$} \\
\hline DE205BAireA/ireA* & ireA Complemented strain & {$[21]$} \\
\hline DE205B $\triangle 0007$ & 0007 Deletion mutant strain & This study \\
\hline DE205B $\triangle 0007 / 0007^{*}$ & 0007 Complemented strain & This study \\
\hline DE205B $\triangle 0008$ & 0008 Deletion mutant strain & This study \\
\hline DE205B $\triangle 0008 / 0008^{*}$ & 0008 Complemented strain & This study \\
\hline DE205B $\triangle 2235$ & 2235 Deletion mutant strain & This study \\
\hline DE205B $\triangle 2235 / 2235^{*}$ & 2235 Complemented strain & This study \\
\hline DE205B $\triangle$ tonB & ton $B$ deletion mutant strain & This study \\
\hline DE205B $\triangle$ tonB/ton $B^{*}$ & tonB Complemented strain & This study \\
\hline $\mathrm{DH} 5 \mathrm{a}$ & Competent invitrogen cells & Vazyme \\
\hline BL21 & Competent invitrogen cells & Vazyme \\
\hline \multicolumn{3}{|l|}{ Plasmid } \\
\hline pKD46 & Amp, expresses $\lambda$ red recombinase & [26] \\
\hline pKD4 & Kan, template plasmid & {$[26]$} \\
\hline pSTV28 & $\mathrm{Cm}$, expression using lac promotor & TaKaRa \\
\hline pCP20 & $\mathrm{Cm}$, Amp, yeast Flp recombinase gene, FLp & {$[26]$} \\
\hline pET32a & Amp, expresses a fusion fragment of the His tag & This study \\
\hline pGEX-4t-1 & Amp, expresses a fusion fragment of the GST tag & This study \\
\hline
\end{tabular}


USA) or solid LB medium supplemented with $2 \%$ agar, followed by incubation in a shaker at $37^{\circ} \mathrm{C}$ and $180 \mathrm{rpm}$ unless otherwise stated. For culture of the mutant strain, appropriate antibiotics (ampicillin, $100 \mu \mathrm{g} / \mathrm{mL}$; kanamycin, $50 \mu \mathrm{g} / \mathrm{mL}$; chloramphenicol, $15 \mu \mathrm{g} / \mathrm{mL}$ ) were added to the LB media. M9 medium was prepared as follows: $200 \mathrm{~mL}$ of $5 \times \mathrm{M} 9$ salt solution $\left(12.8 \mathrm{~g} \mathrm{Na}_{2} \mathrm{PO}_{4} \cdot 7 \mathrm{H}_{2} \mathrm{O}\right.$, $3.0 \mathrm{~g} \mathrm{KH}_{2} \mathrm{PO}_{4}, 0.5 \mathrm{~g} \mathrm{NaCl}$, and $1.0 \mathrm{~g} \mathrm{NH}_{4} \mathrm{Cl}$ ) was mixed with $2 \mathrm{~mL}$ of $1 \mathrm{M} \mathrm{MgSO}_{4}, 20 \mathrm{~mL}$ of $20 \%$ glucose, and $0.1 \mathrm{~mL}$ of $1 \mathrm{M} \mathrm{CaCl}_{2}$. This solution was then dissolved in $1000 \mathrm{~mL}$ double-distilled water and filtered through a $0.22-\mu \mathrm{m}$ membrane. $\mathrm{Fe}\left(0.1 \mathrm{mM} \mathrm{FeCl}{ }_{3} \cdot 6 \mathrm{H}_{2} \mathrm{O}\right)$ was added or not added to M9 medium to establish iron-rich or iron-depleted conditions, respectively.

\section{Construction of the mutant and complemented strains}

For analysis of the role of the four TBDRs in iron uptake, three genes encoding TBDRs $(0007,0008,2235)$ were knocked out, and the ireA mutant strain, which was previously constructed [21], was used in this study. A single mutant strain for each of the three genes $(0007,0008$, 2235) was constructed using the Red homologous recombination method in E. coli [26]. Briefly, for generation of the 0007 mutant strain, a gene-targeting fragment containing a homologous arm on both sides of 0007 was amplified by PCR using the plasmid pKD4 as the template, which contains the phage $\lambda$ Red system under the control of an arabinose promoter. Competent DE205B cells containing $\mathrm{pKD} 46$ were then prepared; $\mathrm{L}$-arabinose was added to induce the expression of the phage $\lambda$ Red system, and the target fragments were transformed by electroporation into DE205B to replace 0007 with the resistance gene. The recombinant strain was screened by growth on LB plates supplemented with kanamycin and identified by crossPCR. Details of the primers (0007-F/0007-R, K1, K2) used for amplification are listed in Additional file 1. Next, the temperature-sensitive plasmid pCP20 was transformed into the recombinant strain to remove the resistance gene. Finally, pCP20 was removed by growth at $42{ }^{\circ} \mathrm{C}$ for $24 \mathrm{~h}$ to obtain the mutant strain DE205B $\triangle 0007$ that did not show any resistance. For analysis of the effect of ton $B$ deletion on the TBDRs, a $\operatorname{ton} B$ mutant strain was also constructed using the same method. Similarly, the 0008, 2235, and ton $B$ mutant strains, namely, DE205B $\Delta 0008$, DE205B $\Delta 2235$, and DE205B $\Delta$ tonB, respectively, were obtained.

The construction of the complemented strain involved recovering the deleted gene. The genomic DNA of DE205B was used as the template to amplify 0007, including its putative promoter gene. The PCR product was then purified and inserted into pSTV28, and the composite vector PSTV28-0007 was transformed by electroporation into $\mathrm{DE} 205 \mathrm{~B} \triangle 0007$ to produce the complemented strain DE205B $\triangle 0007 / 0007^{*}$. In the same manner, the complemented strains DE205B $\triangle 0008 / 0008^{*}$, DE205B $\Delta 2235 / 2235^{*}$, and DE205B $\Delta \operatorname{ton} B / \operatorname{ton} B^{*}$ were obtained. The ireA deletion strain DE205B $\triangle$ ireA and the complemented strain DE205B $\triangle$ ireA/ire $A^{*}$ were constructed in an earlier study and used in this study as well [21].

\section{Growth curves}

Growth curves of the wild-type (WT), mutant (DE205B $\triangle$ ireA, DE205B $\triangle 0007$, DE205B $\triangle 0008$, DE205B $\triangle 2235)$ and complemented strains (DE205B $\Delta$ ireA/ire $A^{*}$, DE205B $00007 / 0007^{*}$, DE205B $\left.\Delta 0008 / 0008^{*}, \mathrm{DE} 205 \mathrm{~B} \Delta 2235 / 2235^{*}\right)$ cultured in LB, iron-depleted M9 and iron-rich M9 media were constructed. All strains were grown overnight in $5 \mathrm{~mL}$ of LB medium supplemented with antibiotics, as appropriate. The cultured strains were centrifuged at $5000 \mathrm{rpm}$ for $10 \mathrm{~min}$ to remove the supernatant, and the cell pellets were washed twice with PBS containing $200 \mathrm{mM} \mathrm{2,2'-dipyridyl;} \mathrm{the} \mathrm{optical} \mathrm{density} \mathrm{at}$ $600 \mathrm{~nm}\left(\mathrm{OD}_{600}\right)$ was adjusted to 1 . Then, $100 \mu \mathrm{L}$ of the suspension was inoculated into $50 \mathrm{~mL}$ of LB medium, and 1000 $\mu \mathrm{L}$ of this solution was inoculated into $50 \mathrm{~mL}$ of iron-depleted or iron-rich M9 medium. The $\mathrm{OD}_{600}$ of the LB culture was measured using a spectrophotometer (Philes, Nanjing, China) every hour for a total of $10 \mathrm{~h}$ and that of the iron-depleted and iron-rich M9 cultures was measured at $0,4,8,12,24$, and $48 \mathrm{~h}$ [27].

\section{Gene expression analyses}

As ireA, 0007 (GI: MN239889), 0008 (GI: MN239890), and 2235 (GI: MN239892) encode putative TBDRs, their expression levels were determined under iron-rich and iron-depleted conditions. DE205B was cultured to the mid-log phase in both types of M9 media, and the gene expression levels were determined using quantitative real-time PCR (qRT-PCR). Briefly, total RNA was extracted from bacteria grown under different culture conditions using a bacterial RNA kit (Omega Bio-Tek, Beijing, China) and reverse transcribed into cDNA using the PrimeScript ${ }^{\mathrm{TM}}$ RT reagent Kit (TaKaRa, Perfect Real Time, Japan) according to the manufacturer's instructions. qRT-PCR was performed in a $20 \mu \mathrm{L}$ reaction volume containing SYBR Green PCR Master Mix (Vazyme Biotech, Nanjing, China) and $0.1 \mu \mathrm{M}$ primers (Additional file 1) specific to the iron uptake-related genes $(0007,0008,2235$, ireA, fecA, fhuA, iutA, iroN1, iroN2); the quantification data were analysed with $\mathrm{ABI}$ StepOne Software, version 2.3 (USA). For analysis of the effect of gene deletion on the ferric uptake system, the expression levels of iron uptake-related genes ( $f e c A$, fhuA, fepA, fepC, feoB, fyuA, iutA, chuA, iroN1, iroN2) were tested in LB medium. Total RNA was extracted from the WT, mutant (DE205B $\triangle$ ireA, DE205B $\triangle 0007$, DE205B $\triangle 0008$, DE205B $\triangle 2235)$, and complemented (DE205B $\Delta$ ireA/ireA*, DE205B ${ }^{*}$ 0007/0007*, 
DE205B $\Delta 0008 / 0008^{*}, \quad$ DE205B $\Delta 2235 / 2235^{*}$ ) strains using the same method. For analysis of the effect of ton $B$ on the expression levels of the genes encoding the four TBDRs, the gene $(0007,0008,2235$, ire $A, f e c A, f h u A$, $f e p A$, iut $A, f e o B$ ) expression levels of the WT strain and DE205B $\Delta$ tonB cultured in iron-depleted M9 medium were examined. Primer details are listed in Additional file 1. dnaE was used as an internal reference gene [21, 22]. The relative gene expression levels were calculated using the $2^{-\triangle \triangle \mathrm{Ct}}$ method; the values are expressed as percentages [28-30].

\section{Expression of the TBDRs}

The pertinent gene fragments (ireA, 0007, 0008, 2235) were inserted into the plasmid pET32a, and ton $B$ was cloned into pGEX-4t-1. Proteins were expressed in BL21 (Vazyme Biotech) via addition of $1 \mathrm{mM}$ isopropyl $\beta$-D-1-thiogalactopyranoside in the mid-log phase. The recombinant proteins IreA, 0007, 0008, and 2235 expressed in vitro carried a His tag, and TonB carried a glutathione-S-transferase (GST) tag. The primer details are listed in Additional file 1. The His-tag fusion proteins were purified by $\mathrm{Ni}$-column chromatography, and the biological activity of the proteins in inclusion bodies was restored via a conventional method that involves using a urea-containing protein refolding solution (including $20 \mathrm{mM}$ Tris-HCl, $1 \mathrm{mM}$ GSH, $0.2 \mathrm{mM}$ GSSG, $0.5 \mathrm{M}$ $\mathrm{NaCl})$. Finally, we concentrated and collected the putative TBDRs (IreA, 0007, 0008, 2235) with His tags by centrifugation in an ultrafiltration tube $(10 \mathrm{kDa})$ at $4{ }^{\circ} \mathrm{C}$. The final fusion proteins were thus obtained $(34 \mathrm{kDa}$ for 0007-His, $66 \mathrm{kDa}$ for 0008-His, $95 \mathrm{kDa}$ for 2235-His, and $93 \mathrm{kDa}$ for IreA-His).

\section{GST pulldown assay}

The functional domain of TonB (residues 150-239) is critical, as it directly interacts with the OM receptor [31]. As such, to further verify whether the four putative TBDRs directly interact with TonB, we performed GST pulldown assays. Cells expressing the GST fusion proteins were lysed using an ultrasonic cell disrupter system (Thermo Fisher Scientific, China). Unlysed cells and impurities were removed by centrifugation $(5000 \mathrm{rpm}$, $10 \mathrm{~min}$ ), and the supernatant was incubated with GSTbinding beads (Enriching Biotechnology, Ltd., Shanghai, China) for $3 \mathrm{~h}$, followed by stringent washing with PBS (2 mM KH $\mathrm{PO}_{4}, 2.6 \mathrm{mM} \mathrm{KCl,} 8 \mathrm{mM} \mathrm{Na} \mathrm{HPO}_{4}, 136 \mathrm{mM}$
$\mathrm{NaCl}$ ) to minimize nonspecific binding. Next, the samples were incubated with the putative TBDRs (IreA, 0007, 0008, 2235) with His tags. Samples were collected after multiple washes with PBS to minimize nonspecific binding. Finally, protein separation was performed by SDSPAGE on $12 \%$ protein gels (Warbio, Shanghai, China), followed by protein detection via a Western blot assay with His-tagged monoclonal antibodies [32, 33]. Further, pGEX-4t-1 (no gene fragment inserted) was incubated with the putative TBDRs, and this was used as the negative control to exclude nonspecific binding of prey proteins to the beads or to GST itself.

\section{Iron uptake test}

To verify whether the four TBDRs are directly involved in iron uptake, we determined the Fe concentrations in the WT, mutant, and complemented strains of DE205B using an iron colorimetric assay kit (Elabscience Biotech, Wuhan, China, catalogue no. E-BC-K139-S). The WT, mutant (DE205B $\Delta$ ireA, DE205B $\Delta$ tonB, DE205B $\Delta 0007$, DE205B $\Delta 0008$, DE205B $\Delta 2235)$, and complemented (DE205B $\Delta$ ireA/ireA*, DE205B $\Delta$ tonB/tonB*, DE205B $\Delta 0007 / 0007^{*}, \quad$ DE205B $\Delta 0008 / 0008^{*}$, DE205B $\left.\Delta 2235 / 2235^{*}\right)$ strains were incubated overnight in $5 \mathrm{~mL}$ of LB medium supplemented with antibiotics, as appropriate. The cultured strains were then centrifuged (5000 rpm, $10 \mathrm{~min}$ ), and the supernatant was discarded. The obtained cell pellets were washed twice with PBS containing 200 M 2,2'-dipyridyl, and the $\mathrm{OD}_{600}$ was adjusted to 1 . Then, $1000 \mu \mathrm{L}$ of the suspension was inoculated into $100 \mathrm{~mL}$ of LB media. Briefly, the strains were grown to the log phase and washed three times with $0.9 \% \mathrm{NaCl}$. Cell pellets were subsequently obtained and suspended in PBS, and the cells were lysed using an ultrasonic cell disrupter system (Thermo Fisher Scientific, USA) to release intracellular iron. Finally, impurities were removed by centrifugation ( $5000 \mathrm{rpm}, 10 \mathrm{~min}$ ); a sample of the supernatant was used for iron content determination [34]. Deionized water $(0.5 \mathrm{~mL})$, iron standard stock solution $(0.5 \mathrm{~mL})$, and sample $(0.5 \mathrm{~mL})$ were individually mixed with a chromogenic agent $(1.5 \mathrm{~mL})$, boiled for $5 \mathrm{~min}$, and then centrifuged at $2300 \mathrm{rpm}$ for $10 \mathrm{~min}$; the supernatant was subsequently collected. According to the manufacturer's instructions for the iron colorimetric assay kit, iron content was estimated by measuring the $\mathrm{OD}$ at $520 \mathrm{~nm}\left(\mathrm{OD}_{520}\right)$ of the supernatant. The following formula was used:

$$
\begin{aligned}
\text { Fe concentration }(\mu \mathrm{g} / \mathrm{L})= & \left(\text { sample } \mathrm{OD}_{520}-\text { deionized water } \mathrm{OD}_{520} / \text { iron standard stock solution } \mathrm{OD}_{520}\right) \\
& - \text { deionized water } \mathrm{OD}_{520} \times \text { standard iron concentration }(2000 \mu \mathrm{g} / \mathrm{L})
\end{aligned}
$$




\section{Statistical analyses}

Statistical analyses were performed in GraphPad Prism 7.0 using unpaired t-tests [35]. In the figures, the error bars indicate the standard deviations, "*" represents $P<0.05$, “*:*" represents $P<0.01$, and “*:**” represents $P<0.001$. qRT-PCR data from three individual experiments were used to determine the differences (fold change) in gene transcription levels. Similarly, in the growth curve and iron uptake analyses, each reaction was performed three times to overcome any experimental errors.

\section{Results}

Iron depletion upregulated the expression levels of the genes encoding the putative TBDRs

The expression levels of all four genes were significantly upregulated under iron-depleted conditions compared with iron-rich conditions; the expression levels of 0007 , 0008 and 2235 were upregulated 1.76 times $(P<0.001)$, 1.43 times $(P<0.05)$, and 1.71 times $(P<0.01)$, respectively. The ire $A$ gene was the most upregulated (1.87 times, $P<0.001$ ) (Figure 1). In addition, the expression levels of the confirmed iron-uptake genes were upregulated under iron-depleted conditions compared to ironrich conditions: the expression levels of $f e c A$ and $f h u A$ were significantly upregulated $(P<0.01)$, but those of $i u t A$, iroN1, and iroN2 were not significantly changed (Figure 1).

\section{Iron depletion decreased the growth rate of the mutant strains}

Growth curves of the WT and mutant strains cultured in LB, iron-depleted M9 and iron-rich M9 media were constructed. The obtained results indicated that the growth rates of the mutant strains (DE205B $\triangle$ ire A,

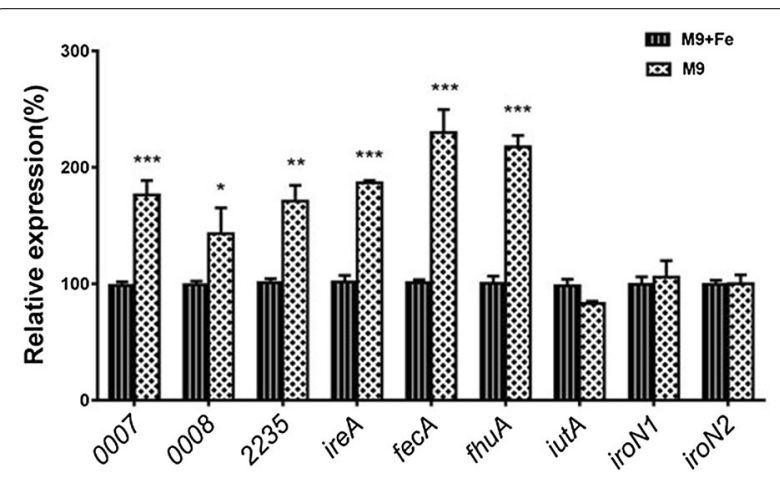

Figure 1 Expression of the iron uptake-related genes in M9 media. The gene expression levels of the iron uptake-related genes in iron-rich or iron-depleted M9 media were tested by qRT-PCR. The relative gene expression levels were calculated using the $2^{-\Delta \Delta c t}$ method; the values are expressed as percentages.
DE205B $\Delta 0007, \mathrm{DE} 205 \mathrm{~B} \Delta 0008$ and DE205B $\Delta 2235)$ were comparable to that of the WT strain in LB medium (Figure 2A). Under iron-depleted conditions, the growth rates of the mutant strains were slightly lower than that of the WT strain (Figure 2B). However, under iron-rich conditions, the growth rates of the mutant strains were comparable to that of the WT strain (Figure 2C).

\section{Compensatory expression of other iron uptake-related} genes was significantly upregulated in the mutant strains Most iron uptake-related genes in DE205B $\triangle$ ire $A$, including $f e c A$, fhu $A, f e p A, f e p C$, feoB, and $f y u A$, were significantly upregulated $(P<0.05)$; the expression levels of all these genes were restored to WT levels in the complemented strain DE205B $\Delta$ ireA/ireA* (Figure 3A). In contrast, relative to those of the WT strain, the expression levels of $f e c A$, chuA, and iroN2 were higher only in DE205B $\Delta 0007, \mathrm{DE} 205 \mathrm{~B} \Delta 0008$, and DE205B $\Delta 2235$, respectively (Figures $3 \mathrm{~B}-\mathrm{D})$.

\section{IreA, 0007, 0008, and 2235 are TBDRs}

Protein-protein interactions between the four putative TBDRs and TonB were analysed in vitro. The SDS-PAGE results showed that these proteins specifically bound to TonB; further, the Western blot assay results revealed the presence of a His tag on the TBDRs. These findings confirmed that TonB positively interacted with all four putative TBDRs (Figures 4A, B).

\section{Iron uptake by DE205B $\triangle$ ireA increased and that by DE205B $\Delta$ ton $B$ decreased}

We found that at the mid-log phase of growth, Fe uptake by DE205B $\triangle$ ire $A$ (Figure $5 \mathrm{~A}$ ) increased $(P<0.05)$ compared to that by the WT strain, whereas Fe uptake by DE205B $\Delta$ tonB (Figure $5 \mathrm{~B}$ ) decreased $(P<0.05)$. Fe uptake by the complemented strains was restored to normal. There were no significant differences in Fe uptake between the 0007, 0008, and 2235 mutant strains and the WT strain (Figures 5D-F). In addition, compared to the medium for the WT and complemented strains, the medium in which DE205B $\Delta$ tonB was cultured turned red (Figure 5C).

\section{The TBDRs are regulated by fur}

The expression levels of the genes encoding the TBDRs were examined by qRT-PCR in the WT strain, $\mathrm{DE} 205 \mathrm{~B} \Delta$ tonB, and DE205B $\Delta$ tonB/tonB* under irondepleted conditions. The ire $A$ expression level in DE205B $\Delta$ ton $B$ was significantly upregulated $(P<0.01)$; furthermore, the expression levels of 0007, 0008, and 2235 were slightly upregulated (Figure 6). In addition, we observed that the expression level of the well-known TBDR fepA was significantly increased 

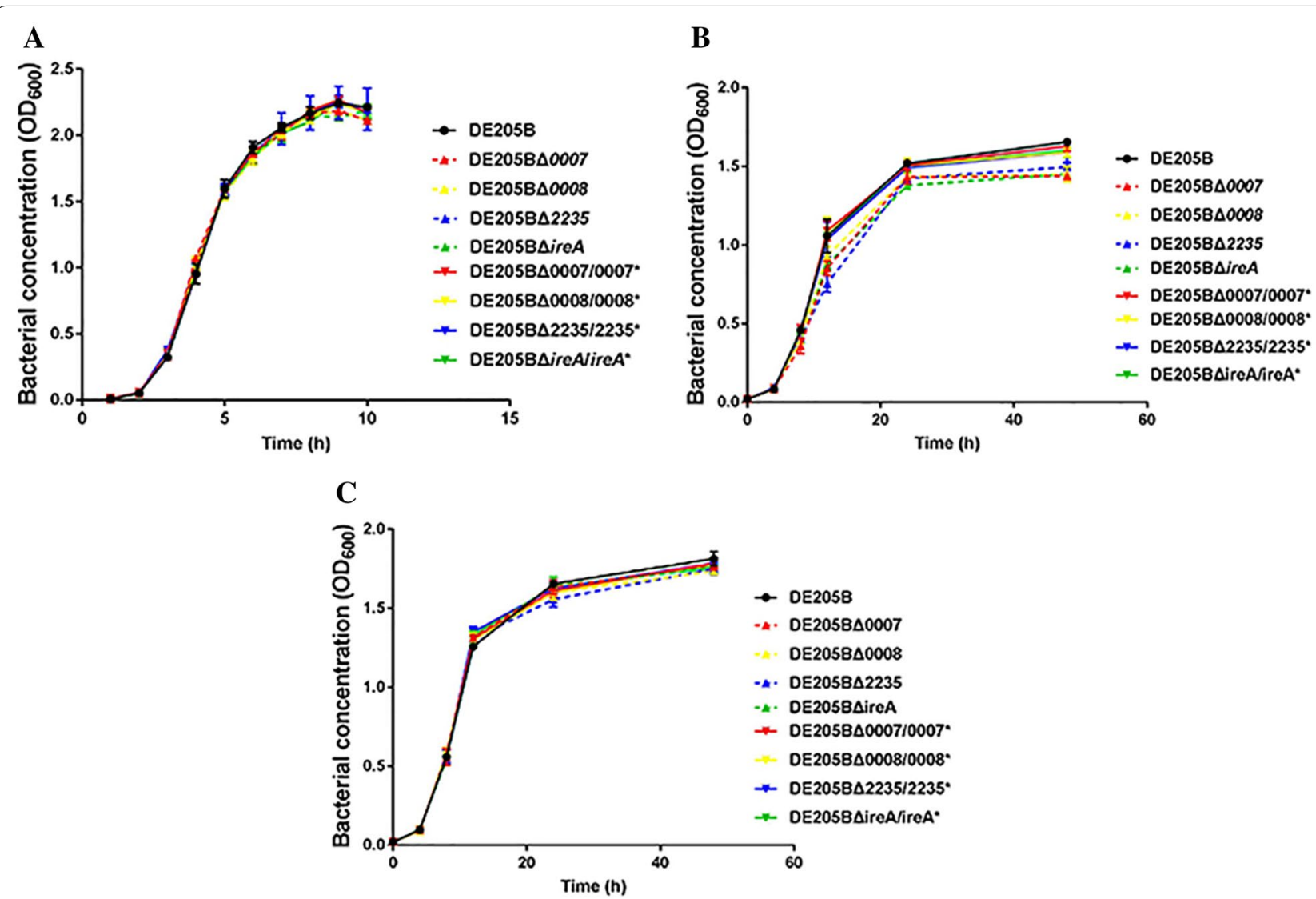

Figure 2 The growth curves of the different strains cultured in LB and iron-depleted M9 media. The growth curves of the WT, mutant

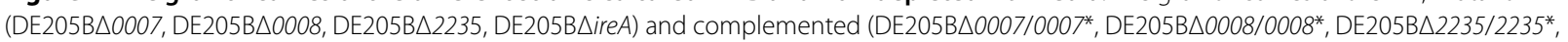
DE205B $\triangle$ ire A/ire $A^{*}$ ) strains cultured in LB, iron-depleted and iron-rich M9 media. Bacterial growth was estimated by measuring the optical density at $600 \mathrm{~nm}\left(\mathrm{OD}_{600}\right)$. A LB medium; $\mathbf{B}$ iron-depleted M9 medium; C iron-rich M9 medium.

$(P<0.01)$, consistent with the results for ireA. Given that the TBDRs are under the control of the ferric uptake regulator fur, the expression levels of fur in the WT strain and DE205B $\triangle$ tonB under iron-depleted conditions were examined. qRT-PCR showed that relative to that of the WT strain, the expression level of fur in DE205B $\Delta$ tonB was downregulated $(P<0.01)$, indicating that the expression of the genes encoding the TBDRs was regulated by fur in the mutant strain DE205B $\Delta$ tonB (Figure 7).

The expression levels of the genes encoding the four TBDRs in DE205B $\triangle$ ton $B$ were further tested under iron-rich or iron-depleted conditions using qRT-PCR. The obtained results indicated that the expression level of ireA was upregulated 6.5 times $(P<0.05)$ under iron-depleted conditions compared to iron-rich conditions and that the expression levels of 0007, 0008, and 2235 were also slightly upregulated (Figure 8). The expression levels of the genes encoding known TBDRs, including $f e p A$, iut $A$, and $f e o B$, were also significantly increased $(P<0.05)$.

\section{Discussion}

Here, six putative TBDRs were predicted, but deletion or mutant strains could be obtained for only four genes encoding TBDRs (ireA, 0007, 0008, 2235). Motif analyses of ireA sequences have helped reveal the $\mathrm{C}$-terminal region of the TBDRs $[12,13]$, but experimental verification is lacking. Herein, IreA and TonB were expressed in vitro, and their interaction was determined via GST pulldown assays, which confirmed that ireA is a TBDR. In addition to ire $A$, three genes (0007, 0008, and 2235) were confirmed to encode TBDRs; however, compared to the expression levels of 0007, 0008, and 2235, that of ireA was the most upregulated in response to iron depletion. Moreover, in the ireA mutant strain $\mathrm{DE} 205 \mathrm{~B} \triangle$ ire $A$, iron uptake was increased, consistent with the excessive compensatory expression of other iron uptake-related genes. These findings are similar to those reported for the known TBDR fepA, indicating that ire $A$ might function similarly to fepA [17].

TBDRs transport ferric siderophores on the $\mathrm{OM}$ with a lack of energy, relying on TonB to transduce energy 

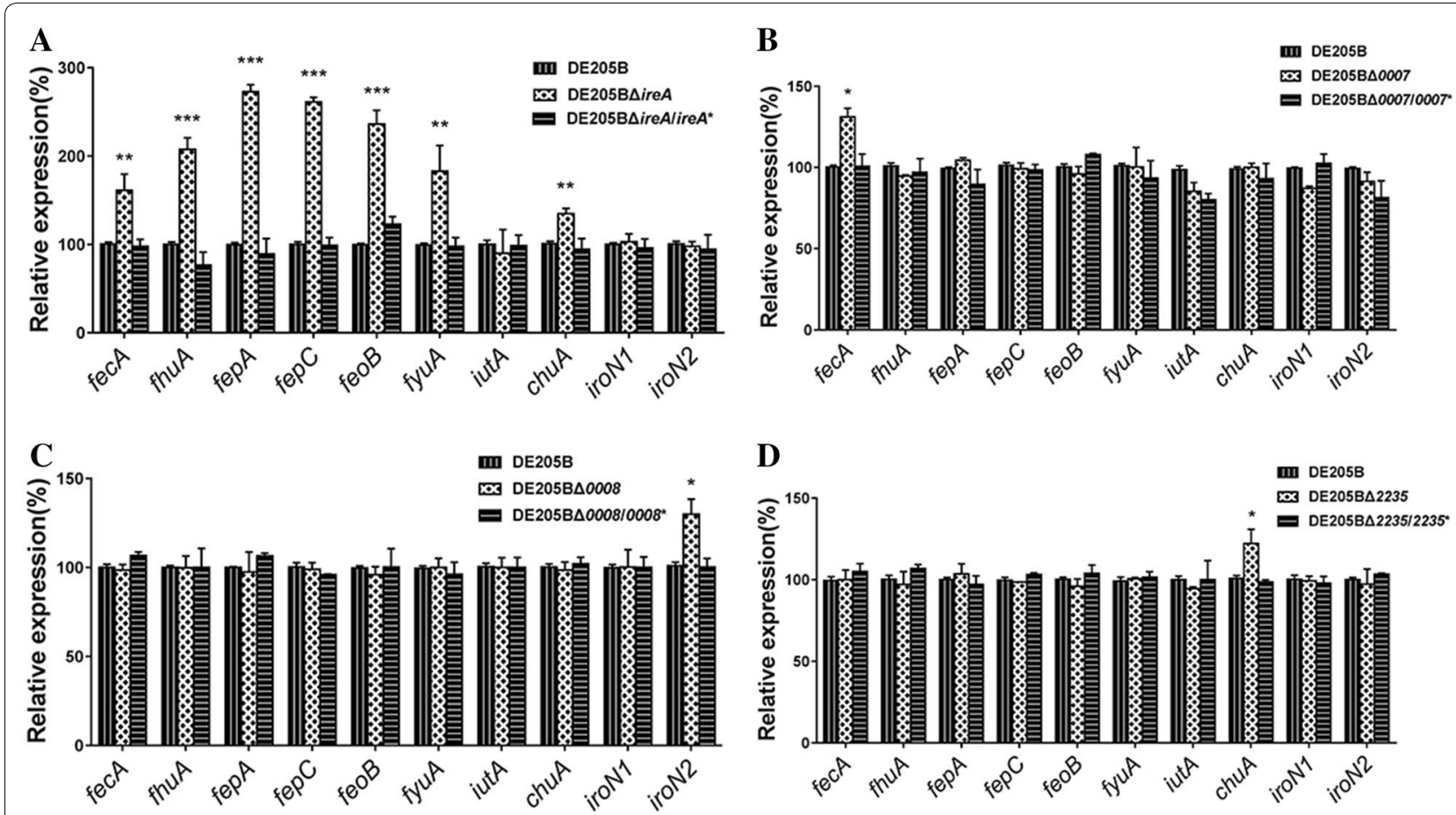

Figure 3 The expression levels of the iron uptake-related genes. The expression levels of the iron uptake-related genes (fecA, fhuA, fepA, fepC, feoB, fyuA, iutA, chuA, iroN1, iroN2) were tested by qPCR. A The expression levels of the iron uptake-related genes in the WT strain, DE205B $\triangle$ ireA, and DE205B $\triangle$ ireA/ire $A^{*}$. B The expression levels of the iron uptake-related genes in the WT strain, DE205B $\triangle 0007$, and DE205B $\triangle 0007 / 0007^{*}$. C The expression levels of the iron uptake-related genes in the WT strain, DE205B $\triangle 0008$, and DE205B $\triangle 0008 / 0008^{*}$. D The expression levels of the iron uptake-related genes in the WT strain, DE205B $\triangle 2235$, and DE205B $2235 / 2235^{*}$. The relative gene expression levels were calculated using the $2^{-\triangle \Delta C t}$ method; the values are expressed as percentages.

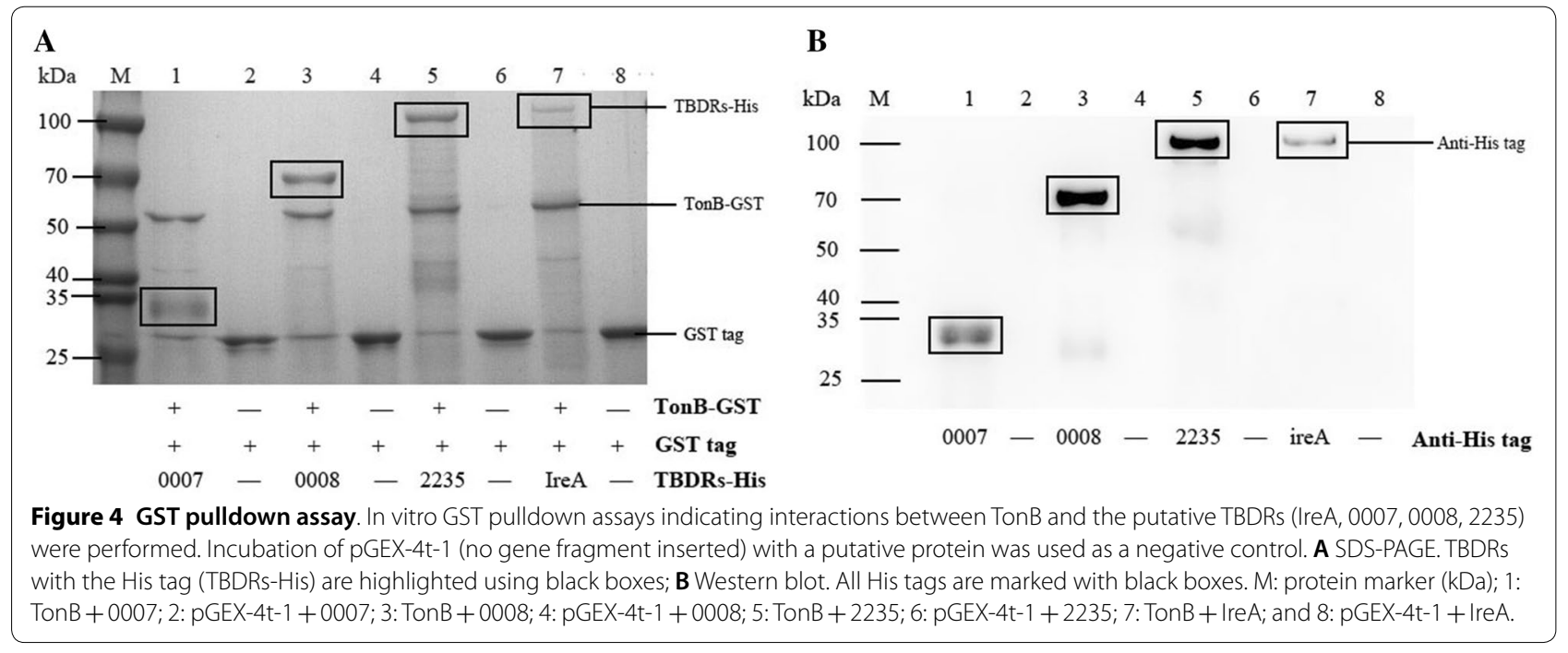

from the proton motive force of the ExbB-ExbD complex in the inner membrane [36, 37]. In this study, a ton $B$ mutant strain was constructed to analyse the effect of ton $B$ deletion on TBDRs. The obtained results indicated that iron uptake by this mutant strain was significantly decreased; the medium used for culturing DE205B $\triangle \operatorname{ton} B$ turned red as the free iron in the culture medium was oxidized. In addition, the expression level of ire $A$ in 

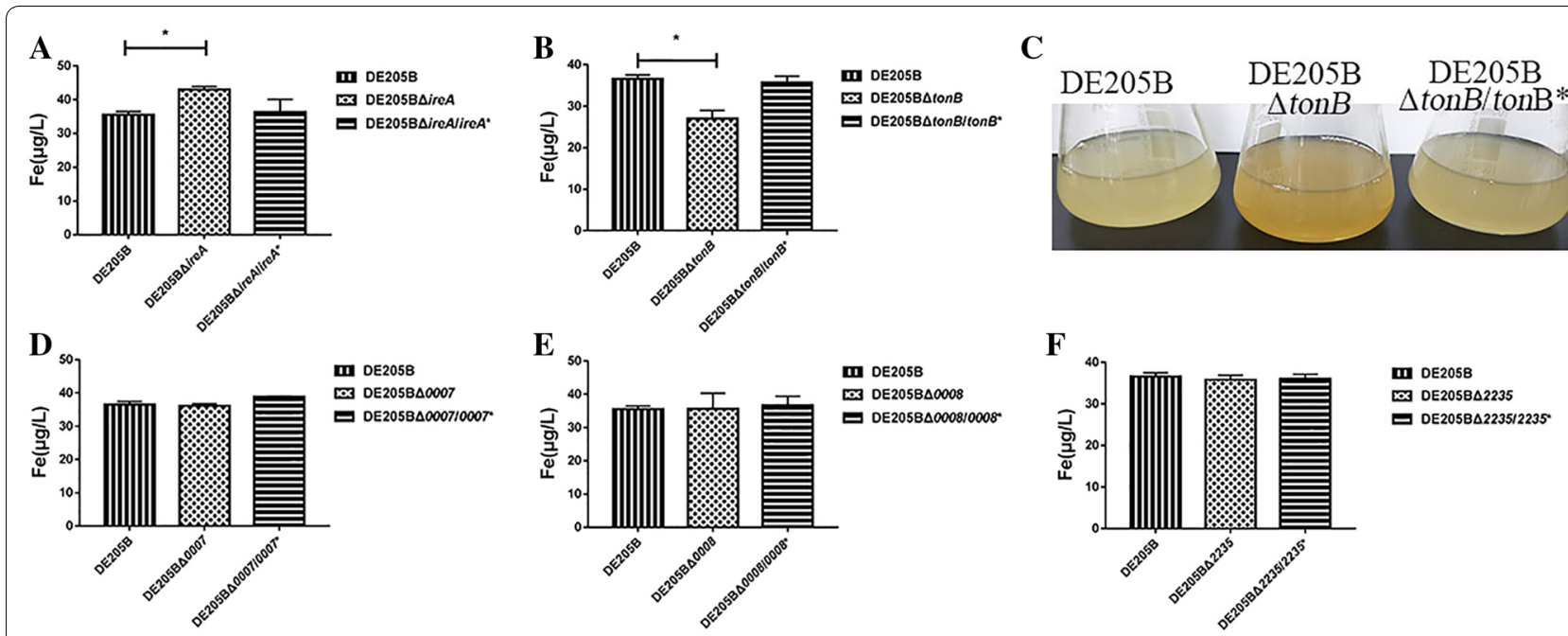

Figure 5 The cellular Fe contents of the WT, mutant, and complemented strains. The different strains were cultured in LB medium to the log phase. The cellular Fe contents of the WT, mutant, and complemented strains were determined using an iron colorimetric assay kit. A The cellular Fe contents of the WT strain, DE205B $\triangle$ ireA, and DE205B $\triangle$ ireA/ireA*; B The cellular Fe contents of the WT strain, DE205B $\triangle$ tonB, and DE205B $\triangle$ tonB/tonB*; C The culture medium used to grow DE205B $\triangle \operatorname{ton} B$ turned red; D The cellular Fe contents of the WT strain, DE205B $\triangle 0007$, and DE205B $\triangle 0007 / 0007^{*}$; E The cellular Fe contents of the WT strain, DE205B $\triangle 0008$, and DE205B $\triangle 0008 / 0008^{*}$; F The cellular Fe contents of the WT strain, DE205B $\triangle 2235$, and DE205B $2235 / 2235^{*}$.

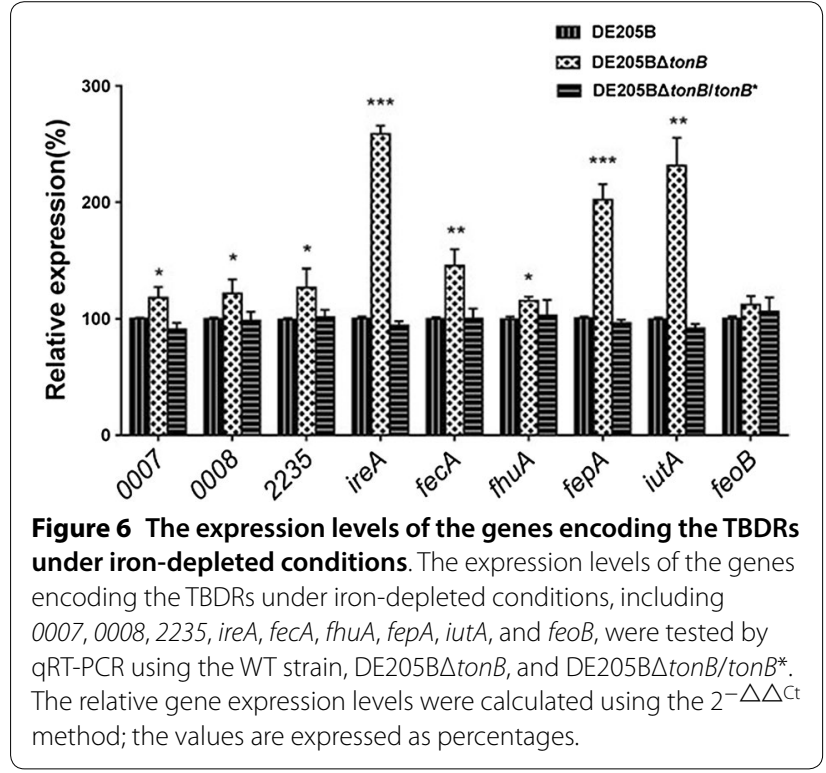

DE205B $\triangle$ tonB was significantly upregulated and that of fur was downregulated. Due to severe iron uptake defects, an iron-depleted environment was created, causing fur to upregulate the expression of the TBDRs in DE205B $\triangle$ tonB, which implies that the TBDRs are regulated by fur and not directly affected by the presence or absence of $\operatorname{ton} B$.

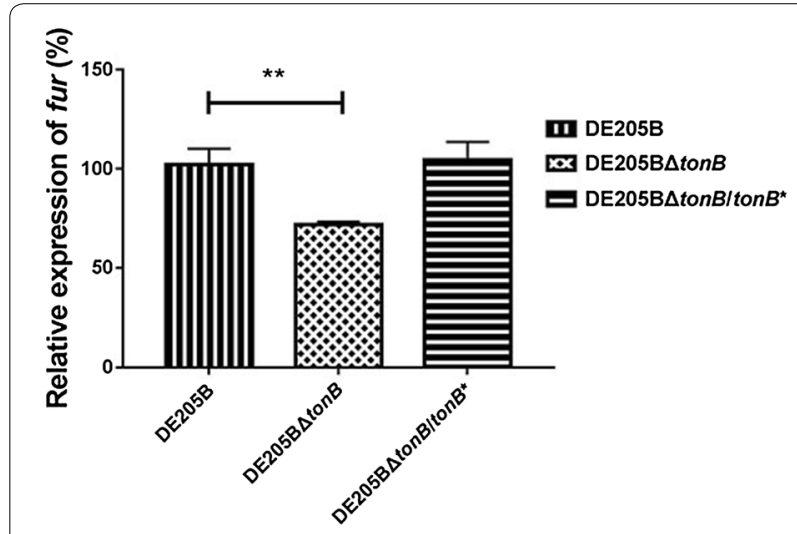

Figure 7 The expression level of fur in iron-depleted M9 media.

The three columns represent the relative gene expression levels

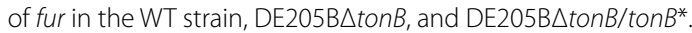
The expression level of fur in the mutant strain was significantly downregulated relative to that of the WT strain $(P<0.01)$.

ireA was initially found in ExPEC, predicted to be a TBDR, and believed to play a role in the colonization of $E$. coli by functioning as a receptor on the OM [13]. In this study, ireA was indeed shown to be a TBDR. Our previous research has proven that ire $A$ also plays a role in adhesion and stress resistance in APEC [13, 21]. Adhesion is the first step in APEC colonization, and the development of a receptor antagonist could protect against the establishment of a bacterial infection. 


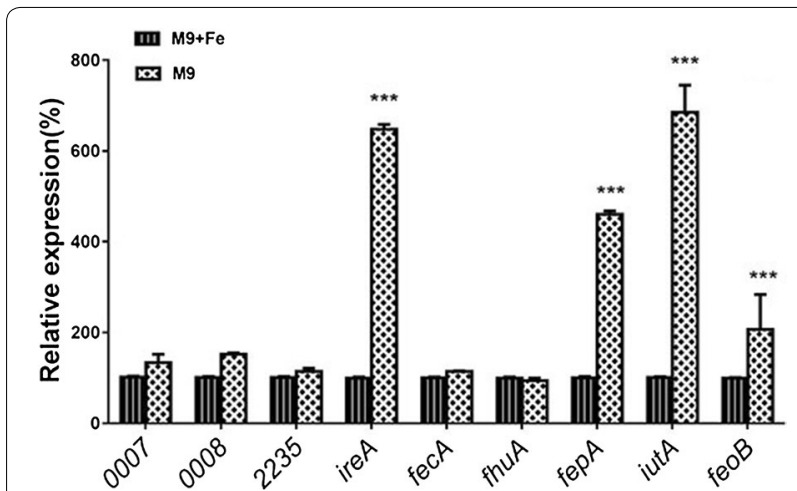

Figure 8 The expression levels of the genes encoding the TBDRs in the mutant strain DE205B $\Delta$ tonB cultured in M9 media. The expression levels of the genes encoding the TBDRs were tested by qRT-PCR under both iron-rich and iron-depleted conditions. The relative gene expression levels were calculated using the $2^{-\triangle \Delta c t}$ method; the values are expressed as percentages.

As a multifunctional receptor, IreA may have potential for vaccine development. Each TBDR is composed of a 22 -strand $\beta$-barrel and an $\mathrm{N}$-terminal TonB box domain with which TonB interacts [38-40]. TonB interacts in vivo with the TonB box of TBDRs (FepA, FhuA, FecA) to form disulphide bonds and eventually transport iron into cells. TBDRs specifically recognize iron carriers. However, the TonB box domain of IreA has not yet been identified, and the iron siderophore structure to which it binds has not been studied either. Future research should focus on investigating these issues.

\section{Supplementary information}

Supplementary information accompanies this paper at https://doi. org/10.1186/s13567-020-0734-z.

Additional file 1: Primers used in this study.

\section{Abbreviations}

APEC: avian pathogenic Escherichia coli; OM: outer membrane; LB: Luria-Bertani medium; $\mathrm{OD}_{600}$ : optical density at $600 \mathrm{~nm}$; qRT-PCR: quantitative real-time polymerase chain reaction; GSH: glutathione, r-glutamyl cysteine + glycine; GSSH: oxidized glutathione; GST: glutathione-S-transferase; WT: wild-type.

\section{Acknowledgements}

We thank Ms. Yaxin Li of Nanjing Agricultural University for providing the ireA deletion mutant.

\section{Authors' contributions}

FT and JD designed the experiments. ZZ wrote the manuscript and performed most of the experiments described in the manuscript. SJ, YL, YS, PY, QG, HZ, and $Y L$ provided help during the experiments. $F X, X Z$, and JR provided valuable suggestions on the manuscript. All authors read and approved the final manuscript.

\section{Funding}

This study was supported by the National Key Research and Development Program of China (2016YFD0500800), the Natural Science Foundation of
Jiangsu Province (BK20180075), and the Fund of the Priority Academic Program Development of Jiangsu Higher Education Institutions (PAPD).

\section{Availability of data and materials}

The datasets analysed during the current study are available upon request from the corresponding author.

\section{Competing interests}

The authors declare that they have no competing interests.

Received: 13 August 2019 Accepted: 11 December 2019

Published online: 23 January 2020

\section{References}

1. Kaper JB, Nataro JP, Mobley HL (2004) Pathogenic Escherichia coli. Nat Rev Microbiol 2:123-140

2. Croxen MA, Finlay BB (2010) Molecular mechanisms of Escherichia coli pathogenicity. Nat Rev Microbiol 8:26-38

3. Markland SM, LeStrange KJ, Sharma M, Kniel KE (2015) Old friends in new places: exploring the role of extraintestinal E. coli in intestinal disease and foodborne illness. Zoonoses Public Health 62:491-496

4. Rodriguez-Siek KE, Giddings CW, Doetkott C, Johnson TJ, Fakhr MK, Nolan LK (2005) Comparison of Escherichia coli isolates implicated in human urinary tract infection and avian colibacillosis. Microbiology 151:2097-2110

5. Manges AR (2016) Escherichia coli and urinary tract infections: the role of poultry-meat. Clin Microbiol Infect 22:122-129

6. Tivendale KA, Logue CM, Kariyawasam S, Jordan D, Hussein A, Li G, Wannemuehler Y, Nolan LK (2010) Avian-pathogenic Escherichia coli strains are similar to neonatal meningitis E. coli strains and are able to cause meningitis in the rat model of human disease. Infect Immun 78:3412-3419

7. Ewers C, Antao EM, Diehl I, Philipp HC, Wieler LH (2009) Intestine and environment of the chicken as reservoirs for extraintestinal pathogenic Escherichia coli strains with zoonotic potential. Appl Environ Microbiol 75:184-192

8. Li G, Laturnus C, Ewers C, Wieler LH (2005) Identification of genes required for avian Escherichia coli septicemia by signature-tagged mutagenesis. Infect Immun 73:2818-2827

9. Dho-Moulin M, Fairbrother JM (1999) Avian pathogenic Escherichia coli (APEC). Vet Res 30:299-316

10. Andrews SC, Robinson AK, Rodriguez-Quinones F (2003) Bacterial iron homeostasis. FEMS Microbiol Rev 27:215-237

11. Huynh C, Andrews NW (2008) Iron acquisition within host cells and the pathogenicity of Leishmania. Cell Microbiol 10:293-300

12. Garenaux A, Houle S, Folch B, Dallaire G, Truesdell M, Lepine F, Doucet N, Dozois CM (2013) Avian lipocalin expression in chickens following Escherichia coli infection and inhibition of avian pathogenic Escherichia coli growth by Ex-FABP. Vet Immunol Immunopathol 152:156-167

13. Russo TA, Carlino UB, Johnson JR (2001) Identification of a new ironregulated virulence gene, ireA, in an extraintestinal pathogenic isolate of Escherichia coli. Infect Immun 69:6209-6216

14. Khan A, Singh P, Srivastava A (2018) Synthesis, nature and utility of universal iron chelator-siderophore: a review. Microbiol Res 212-213:103-111

15. Kim S, Lee JH, Seok JH, Park YH, Jung SW, Cho AE, Lee C, Chung MS, Kim KH (2016) Structural basis of novel iron-uptake route and reaction intermediates in ferritins from gram-negative bacteria. J Mol Biol 428:5007-5018

16. Koster W (2001) ABC transporter-mediated uptake of iron, siderophores, heme and vitamin B12. Res Microbiol 152:291-301

17. Gresock MG, Postle K (2017) Going outside the TonB box: identification of novel FepA-TonB interactions in vivo. J Bacteriol 199:e00649-16

18. Cascales E, Buchanan SK, Duche D, Kleanthous C, Lloubes R, Postle K, Riley M, Slatin S, Cavard D (2007) Colicin biology. Microbiol Mol Biol Rev 71:158-229

19. Schalk IJ, Mislin GL, Brillet K (2012) Structure, function and binding selectivity and stereoselectivity of siderophore-iron outer membrane transporters. Curr Top Membr 69:37-66

20. Gomez-Santos N, Glatter T, Koebnik R, Swiatek-Polatynska MA, SogaardAndersen L (2019) A TonB-dependent transporter is required for secretion 
of protease PopC across the bacterial outer membrane. Nat Commun 10:1360

21. Li Y, Dai J, Zhuge X, Wang H, Hu L, Ren J, Chen L, Li D, Tang F (2016) Ironregulated gene ireA in avian pathogenic Escherichia coli participates in adhesion and stress-resistance. BMC Vet Res 12:167

22. Wang S, Niu C, Shi Z, Xia Y, Yaqoob M, Dai J, Lu C (2011) Effects of ibeA deletion on virulence and biofilm formation of avian pathogenic Escherichia coli. Infect Immun 79:279-287

23. Wang S, Xia Y, Dai J, Shi Z, Kou Y, Li H, Bao Y, Lu C (2011) Novel roles for autotransporter adhesin AatA of avian pathogenic Escherichia coli: colonization during infection and cell aggregation. FEMS Immunol Med Microbiol 63:328-338

24. Wang S, Shi Z, Xia Y, Li H, Kou Y, Bao Y, Dai J, Lu C (2012) IbeB is involved in the invasion and pathogenicity of avian pathogenic Escherichia coli. Vet Microbiol 159:411-419

25. Zhuge X, Wang S, Fan H, Pan Z, Ren J, Yi L, Meng Q, Yang X, Lu C, Dai J (2013) Characterization and functional analysis of AatB, a novel autotransporter adhesin and virulence factor of avian pathogenic Escherichia coli. Infect Immun 81:2437-2447

26. Datsenko KA, Wanner BL (2000) One-step inactivation of chromosomal genes in Escherichia coli K-12 using PCR products. Proc Natl Acad Sci U S A 97:6640-6645

27. Holden KM, Browning GF, Noormohammadi AH, Markham PF, Marenda MS (2012) TonB is essential for virulence in avian pathogenic Escherichia coli. Comp Immunol Microbiol Infect Dis 35:129-138

28. Ali-Benali MA, Alary R, Joudrier P, Gautier MF (2005) Comparative expression of five Lea Genes during wheat seed development and in response to abiotic stresses by real-time quantitative RT-PCR. Biochim Biophys Acta 1730:56-65

29. Livak KJ, Schmittgen TD (2001) Analysis of relative gene expression data using real-time quantitative $P C R$ and the $2^{-\triangle \Delta C t}$ method. Methods 25:402-408

30. Wasilewska I, Gupta RK, Palchevska O, Kuznicki J (2019) Identification of zebrafish calcium toolkit genes and their expression in the brain. Genes 10:E230
31. James KJ, Hancock MA, Gagnon JN, Coulton JW (2009) TonB interacts with BtuF, the Escherichia coli periplasmic binding protein for cyanocobalamin. Biochemistry 48:9212-9220

32. Lu L, Tai G, Hong W (2005) Interaction of Arl1 GTPase with the GRIP domain of golgin-245 as assessed by GST (glutathione-S-transferase) pull-down experiments. Methods Enzymol 404:432-441

33. Kanno E, Ishibashi K, Kobayashi H, Matsui T, Ohbayashi N, Fukuda M (2010) Comprehensive screening for novel rab-binding proteins by GST pull-down assay using 60 different mammalian Rabs. Traffic 11:491-507

34. Ajiboye TO, Skiebe E, Wilharm G (2018) Contributions of ferric uptake regulator Fur to the sensitivity and oxidative response of Acinetobacter baumannii to antibiotics. Microb Pathog 119:35-41

35. Ling D, Salvaterra PM (2011) Robust RT-qPCR data normalization: validation and selection of internal reference genes during post-experimental data analysis. PLoS One 6:e17762

36. Devanathan S, Postle K (2007) Studies on colicin B translocation: fepA is gated by TonB. Mol Microbiol 65:441-453

37. Krewulak KD, Vogel HJ (2011) TonB or not TonB: is that the question? Biochem Cell Biol 89:87-97

38. Sarver JL, Zhang M, Liu L, Nyenhuis D, Cafiso DS (2018) A dynamic protein-protein coupling between the TonB-dependent transporter FhuA and TonB. Biochemistry 57:1045-1053

39. Wang Y, Chen X, Hu Y, Zhu G, White AP, Köster W (2018) Evolution and sequence diversity of FhuA in Salmonella and Escherichia. Infect Immun 86:e00573-18

40. Chimento DP, Kadner RJ, Wiener MC (2005) Comparative structural analysis of TonB-dependent outer membrane transporters: implications for the transport cycle. Proteins 59:240-251

\section{Publisher's Note}

Springer Nature remains neutral with regard to jurisdictional claims in published maps and institutional affiliations.
Ready to submit your research? Choose BMC and benefit from:

- fast, convenient online submission

- thorough peer review by experienced researchers in your field

- rapid publication on acceptance

- support for research data, including large and complex data types

- gold Open Access which fosters wider collaboration and increased citations

- maximum visibility for your research: over $100 \mathrm{M}$ website views per year

At BMC, research is always in progress.

Learn more biomedcentral.com/submissions 